\title{
XIII.
}

\section{Bericht über die Verhandlungen der otologischen Section auf der 74. Versammlung deutscher Naturforscher und Aerzte in Karlsbad vom 21. bis 27. September 1902. \\ Von \\ Dr. Walther Sehnlze und Dr. E. Buhe.}

(Mit 2 Abbildungen.)

Einfübrende: Dr. Hnilits ch ka (Neudeok) und Privatdocent Dr. Piffl (Prag).

Erste Sitzung: Montag den 22. September 1902, Nachmittags $3 \mathrm{Uhr}$.

Vorsitzender: Bezold (Munchen).

Nach Begrüssung der Versammlung dureh Hnilitsehka wird vor Eintritt in die Tagesordnung tiber folgenden von der Abtheilung 21 a (Hals- und Nasenkrankbeiten) und von der Berliner laryngologischen Gesellschaft gestellten Antrag berathen:

„Die Geschäftsleitung der Versammlung deutseher Naturforscher und Aerzte ist zu ersuchen, die diesmal wiederhergestellte Selbständigkeit der beiden Seetionen für Otologie und Laryngologie zu einer dauernden zu gestalten, dagegen die Abtheilungsvorstände zu veranlassen, für die gemeinsam interessirenden rhinologischen und pharyngologisehen Themata gemeinsame Sitzungen zu veranstalten."

Der Antrag wird nach längerer Discussion, an weleher sich Hey mann (Berlin), Müller (Karlsbad), Barth (Leipzig), Kümmel (Breslau), Zaufal (Prag), Berthold (Königsberg i. Pr.), Bezold(Münohen), Ha b erm a n (Graz) betheiligen, angenommen.

Die Theilung gilt vorlậufig nur für die 74. Versammlung deutscher Naturforseher und Aerzte. Bezïglich der gewünschten definitiven Trennung wird in der nächsten Aussehusssitzung der Gesellschaft entsehieden werden. 
74. Versamml. dentsch. Naturforscher u. Aerzte in Karlsbad (otol. Sect.). 105

\section{Vorträge:}

1. Urbantschitsch (Wien): Ueber die elektrokatalytische Behandlung des 0hres.

Urbantsobitseb behandelt nach ehronischem Katarrh oder nach Eiterungen zurüokgebliebene Sehwerhörigkeit unter Benutzung der katalytisehen Wirkung des elektrisehen Stromes, indem er kleine, mit Watte umwickelte Elektroden an die Labyrinthwand oder an das Trommelfell ansetzt und den Strom in einer Stärke von 0,1-0,2 Milliampère 5-15 Minuten einwirken lässt. Es soll danach subjective Erleichterung und meist sofortige Hörverbesserung eintreten.

\section{Discussion:}

Barth (Leipzig): Bei einseitiger elektrischer Behandlung können leicht Schmerzen und Schwindel eintreten. Er giebt deshalb den Rath, den positiven Pol zu theilen und den Strom durch beide Ohren hindurchanleiten. Die Hörverbesserung beruht zum Theil darauf, dass die Elektrode gleichzeitig als künstliches Trommelfell wirkt.

K ü m mel (Breslau) empfiehlt die Anwendung sehr schwacher Ströme und hält die Elektrolyse bei den versehiedensten Ohraffeotionen für äusserst wirksam.

Berthold (Königsberg) hat selbst bei sohwachen Strömen Schwindel beobachtet.

Urbantschitsch (Wien) hält an seiner Methode der katalytischen Behandlung fest und ist gegen die von Barth empfohlene Trennung der Elektrode. Eine Function der Elektrode als künstliches Trommelfell kommt nicht in Frage.

2. Habermann (Graz): Zur Entstehung von Taubstummheit in Folge von Mittelohrerkrankung (publicirt im LVII. Bd. S. 79 dieses Arehivs).

Habermann berichtet über einen Fall von Taubstummheit ohne Erkrankung des Labyrinths und des Nervus acusticus. Die Untersuchung des Gehörorgans ergab Narben im Trommelfell, Verdickung der Paukenschleimhaut, Fixation des Steigbuigels im ovalen Fenster, Verschluss der Nische des runden Fensters durch Hyperostose. In derartigen Fallen verspricht sich Habermann von operativen Eingriffen an den Labyrinthfenstern Erfolg.

\section{Diseussion:}

Gomperz (Wien) glaubt sich in einem Falle überzengt zu. haben, dass sich die Substantia propria namentlich bei kleinen Trommelfellperforationen regeneriren kann. 
Alexander (Wien) führt einen von Politzer beobachteten Fall an, bei welchem normales Gehör bestand trotz des hyperostotischen Verschlusses des run den Fensters. Die Membran des runden Fensters und das Labyrinth waren normal.

Bezold (München) betont, dass auch bei knöcherner Fixation des Steigbügels gutes Gehör vorhanden sein kann, wenn nämlich das runde Fenster normal ist.

Habermann (Graz) sohliesst sich der Ansicht von Bezold an. Es handelt sich in seinen Fällen um hyperostotische Vorgänge, nicht um Sklerosen.

3. Wittmaack (Breslau): Demonstration histologischer Veränderungen am Ganglion spirale bei Chininvergiftung.

Wittmaack demonstrirt mikroskopische Präparate von Ganglienzellen des Ganglion spirale, welehe bei mit Chinin vergifteten Thieren deutliche Veränderungen im Verhalten der $\mathrm{N}$ issl'schen Granula beim Vergleich mit den Ganglienzellen eines normalen Controlthieres erkennen lassen. Dieselben charakterisiren sich in leichten Fällen durch gesteigerte Affinität der Granula zum alkalischen Farbstoff, durch Veränderungen in der Lagerung der einzelnen Granula und in hochgradigen Fällen durch fast gänzliches Ausfallen derselben. (Autoreferat.)

\section{Discussion:}

Löwe (Berlin) hat bei Kaninchen nach Vergiftung mit Morphium analoge Veränderungen an den Ganglienzellen gefunden.

4. Alt (Wien): Ueber Hörnervenerkrankung durch Nicotin und Alkobol.

Der Vortragende berichtet zunäehst über zwei von ihm beobachtete Fälle von Hörnervenerkrankung nach internem Gebrauch von Natrium salicylicum, welche unter Ménière'schen Symptomen verliefen, sowie ubber eine complete Taubheit dureh Chiningenuss während einer Malariaerkrankung. Von Intoxicationsneuritiden durch Alkohol und Nicotin beobachtete Alt 3 Fälle. Bei einem Patienten mit alkoholiseher Polyneuritis und Neuritis optica retrobulbaris trat eine Acnsticuserkrankung (mit dem typischen Befunde einer Labyrinthaffection) auf, die nach stricter Abstinenz in 2 Monaten zuritckging. Ferner 2 Fälle von Nicotinneuritis des Hörnerven mit hochgradiger Schwerhörigkeit und Ohrensausen (hohes Pfeifen) bei negativem Stimmgabelbefunde. Der eine Fall wurde zunäehst unrichtig gedeutet, die 
locale Behandlung blieb ohne Erfolg, die Schwerhörigkeit war progressiv, bis das ätiologische Moment, übermässiges Rauchen, ermittelt wurde, worauf alle Symptome binnen 6 Wochen, ohne jede weitere Behandlung, bei vollständiger Abstinenz zurückgingen. Der Vortragende verweist darauf, dass die Acusticusneuritis durch Nicotin und Alkohol im Anfangsstadium wegen des negativen Stimmgabelbefundes nicht erkannt werde und deshalb zu irreparabeln Läsionen führe, die dann als Labyrintherkrankungen aus unbekannter Ursache gelten. Auch bei der Schwerhörigkeit ex professione ist im Anfangsstadium der Stimmgabelbefnnd negativ, und doch würde das Wesen der Erkrankung mit Rukcksicht auf den lärmenden Beruf rechtzeitig erkannt. Die Acusticuserkrankung tritt meist selbständig ohne Mitbetheiligung des Opticus auf, ebenso wie durch eine Infeetionskrankheit verschiedene isolirte Neuritiden bedingt sein können. Bei der Neuritis acustici ist der Stimmgabelbefund meist negativ, analog dem negativen Spiegelbefund bei der Neuritis retrobulbaris, dem centralen Skotom entspricht das subjective Geräusch (hohes Pfeifen), der Sehschwäche die Herabsetzung des Gehörs. Nur völlige Abstinenz führt zur Heilung, die nieht vor 6 bis 8 Wochen zu erwarten ist. Die Einschränkung des Tabak- bezw. Alkoholgenusses ist nicht ausreichend, da sehon minimale Mengen den erkrankten Nerven in seinem Zustand erhalten. (Autoreferat.)

\section{Discussion:}

Go mperz (Wien): Die in Rede stehenden Affectionen sind nicht zu verwechseln mit katarrhalischen Erkrankungen des Mittelohres, wie sie bei starken Rauchern vorkommen. In schweren Fällen ist durch die Stimmgabeluntersuchung immer eine Schädigung der schallpereipirenden Elemente nachweisbar. Die Ursache der Vergiftung bildet weniger das Nieotin selbst, als dessen Zersetzungsproducte. Völlige Abstinenz ist von günstigem Erfolge.

Habermann (Graz) hat in Fällen von Alkobolvergiftung eine Betheiligung des Labyrinths dureh die Stimmgabeluntersuchung festgestellt. Er sah nach Aspiringebraweh Obrensausen eintreten, welches wieder versehwand, als das Medicament ausgesetzt wurde.

Berthold (Königsberg) Ist der Ansicht, dass geringe Grade von Amblyopie bezw. Sehwerhörigkeit bei Rauchern nicht immer auf einer Neuritis des Nerrus optieus bezw. acusticus beruhen. 
Alt (Wien) giebt zu, dass der ophthalmoskopische Befund oft im Stiche lässt, dass aber andererseits auch mehrfach Atrophie des Nerven und interstitielle Entzündung festgestellt worden ist.

Zweite Sitzung: Dienstag den 23. September Vormittags $91 / 2 \mathrm{Uhr}$.

Vorsitzender: Schwartze (Halle a. S.).

1. Bezold (München): Allgemeine Sepsis bei chronischer Mittelohreiterung mit central gelegener Trommelfellperforation.

Bezold will für die nachfolgenden Fälle, die er zu beobachten Gelegenheit hatte, der Statistik wegen, die Aufstellung einer besonderen Kategorie von Mittelohreiterung befürworten, da man sie weder zu den acuten Mittelohreiterungen rechnen könne, weil es sich nur um Recidive einer chronischen Eiterung handle, noch zu den chronischen.

1. Fall. 15 jăbriges Mädchen, 1893 ambulatorisch behandelt. Seit zwei Jahren wiederholt controlirt. Ausfluss hat aufgehört. Links centralgelegene ovale Oeffnung. 1895 Schmerzen im linken Ohr, sehr heftig, Sensorium benommen, Belag anf den Mandeln, starke Drüsenschwellung, continuirliches hohes Fieber bis über $41^{\circ}$, Erbrechen. Druckempfindlichkeit über dem Warzenfortsatz. Eröffnung des Antrums: Schleim in den Zellen, fibrinöses Exsudat, in demselben Streptokokken. Am andern Tage Sensorium frei, Puls stark beschleunigt, Milzschwellung. Eiweiss im Harn, blutiges Sputum. In den zächsten 'Tagen letaler Ausgang.

Section: Sepsis.

Während der Verlauf dem der acuten Fälle ähnlich ist, lässt der Befund auf einen ohronischen Fall schliessen, sowohl der Zustand des Trommelfelles wie der Befund an der Schleimhaut sprechen für eine chronisohe Erkrankung.

2. F a ll. 23 jähriger Student, früber bereits an einer chronischen Mittelohreiterung behandelt. Unter schweren Allgemeinerscheinungen (Benommenheit, hohes Fieber) wurde er in die Klinik aufgenommen. Es fand sich im Trommelfell eine central gelegene, längs-ovale Perforation. Antrumeröffiunng. Im Eiter Streptokokken. Section ergab Sepsis.

Section des linken Schläfenbeins: Im Trommelfell centrale Perforation von $1 \frac{1}{1} / 2 \mathrm{~mm}$ im Durchmesser. Im Bulbus frisches Gerinnsel. Im Processus mastoideus eitriges Secret. - Zwischen Dura und Felsenbein Eiter, Dura verdickt, Pachymeningitis purulenta circumscripta. Leichte Phlebitis ohne Thrombose im Sinus, Sepsis. Hier waren die Wege deutlicher, aut dem die Infection den Sinus erreicht batte (besser als im 1. Fall). Am Tegmen sowie zwischen Flexur und Knochen Eiter bezw. fibrinöses Exsudat. Die Venenwände zeigten frische fleckige Iujection. Keine Thrombose oder Metastase.

3. Fall. Student, 21 Jahre alt. Beim Baden war Wasser in's Ohr gekommen, nach 5 Tagen Schmerzen, Fieber. Links Secret im Ohr, linsengrosse Perforation im hinteren unteren Quadranten. Druckemptindlichkeit des Warzenfortsatzes. Eröffnung desselben. Keine Streptokokken im Eiter, sondern Stäbchen ähnlich den Influenzastäbchen (diese waren auch in dem vorigen Falle gefunden). Nach der Operation noch 14 Tage Schmerzen, nach 2 Monaten geheilt mit bestehender Perforation und mässiger Secretion entlassen. 
Nach dem Trommelfell- und dem Mittelohrbefund und den sehweren Allgemeinerseheinungen in diesen Fällen muss angenommen werden, dass eine Infection eigener Art vorliegt. Be zold möchte die Erkrankungsform als acute Sepsis bei centralgelegener Perforation bezeichnen. Da die Falle selten sind, glaubt Bezold sie als eine gesonderte Art der Infection auffassen zu milssen. Es müsse sich dabei entweder um eine starke Virulenz oder um eine besonders massenhafte Einwanderung von Mikroorganismen handeln. Der ganze Krankheitsbefund macht es wahrscheinlich, dass eine gleichzeitige Infection sowohl der Mittelohrschleimhant, als auch des Lymphdrüsenapparates vorliege.

\section{Discussion:}

B arth (Leipzig) möehte davor warnen, zu sehr zu sehematisiren. Es gäbe oft Fälle, die zwar chroniseher Natur seien, aber das Bild der acuten Entzündung bieten. Es komme auch sonst bei vielen Krankheiten vor, dass die Infectionsquelle verheilt und trotzdem später sehwere Allgemeinerseheinungen auftreten. Barth hat in Breslau einen Fall gesehen, bei dem eine acute Mittelohrentzündung zurückgegangen war, und der dann an einer aouten Meningitis zu Grunde ging. Am Trommelfell waren kaum noch Erseheinungen einer früheren Erkrankung nachzuweisen. Drüsenschwellungen seien niehts Charakteristisches, dieselben finde man auch sonst sehr häufig.

Kü $\mathrm{mmel}$ (Breslau) weist darauf hin, dass in der Gegend der Tubenmündung und am Boden der Paukenhöhle kleine Schleimhautleisten sich finden. An diesen Stellen könne es leieht zu einer Infeetion des Canalis earotieus oder des Bulbus venae jugularis kommen. Er glaubt, dass dieser Weg der Infeetion gar nicht so selten sei.

Habermann (Graz) hat aeute Sepsis früher of nach Operationen im Spital gesehen. Er glaubt, dass es sich in den angeführten Fällen auch um eine solehe Spitalinfeetion handeln könne.

Schwartze (Halle) meint, es müsse deshalb schon noch dringender die Forderung gestellt werden, dass die Obrenkranken in ein besonderes Krankenhaus kommen.

Grunert (Halle) ist der Ansicht, dass dieselben acuten Erkrankungen, wie sie Bezold gesehildert hat, aueh bei randständiger Perforation vorkommen. Die Lage der Perforation gebe keinen Anhaltspunkt für die Sonderstellung der Fälle. 
Was den Weg der rom Ohr ausgehenden Allgemeininfection betreffe, so darf man nicht immer nur an die Lymphbahnen denken, worauf Leutert zuerst aus der Halle'sehen Klinik nachdrücklich hingewiesen hat auf Grund positiver anatomiseher Befunde. Die Ursache der Allgemeinerkrankung sei eine Thrombophlebitis. Die Erkrankungsformen zeigten bald mehr pyämisohen, bald mehr septischen Charakter.

Biehl (Wien) hat auch Fälle beobachtet, wo keine äusseren Erseheinungen einer stattgehabten Infection mehr vorhanden waren und sehwere septische Allgemeinerseheinungen auftraten. Die Leutert'sche Diagnosenstellung biete für die praktisehen Aerzte eine klare Eintheilung.

Bezold (München) betrachtet seine Fälle hauptsächlich vom statistischen Standpunkte. Es handelt sich weder um acute noch um chronische Fälle, deshalb habe er sie gesondert aufgeführt. Als Spitalinfection kann er die Erkrankungsform nicht auffassen, weil die Fäle schon mit sehweren Erkrankungserseheinungen in die Klinik aufgenommen waren. Es war eine frische Lymphangitis in den Fällen vorhanden, darauf habe er hinweisen wollen, keine einfache Drüsenschwellung.

Alexander (Wien) glaubt, die Ursache sei nur in der Sehwere der Infection zu suehen. Es müsse deshalb in solchen Fällen Eiter und Blut culturell untersucht werden.

Sehwartze (Halle) theilt mit, dass septische Processe vorwiegend bei acuten Fällen anftreten. Es kommt dabei oft nicht zu Metastasen, weil der Krankheitsverlauf zu sehnell zum Tode führt. Entscheidend für die Prognose ist der Allgemeinzustand, vor Allem der Puls, nicht der locale Befund am Ohr.

2. Grunert (Halle): Ueber die nenen'Angriffe gegen die Paracentese des Trommelfelles. (Erscheint ausführlich in der Münchner med. Wochenschrift.)

Ausgehend von dem häufigen Weehsel theoretiseher Anschaungen, denen zu Liebe die praktisohe Medioin niemals in in der Praxis bewährte therapeutische Maassnahmen aufgeben darf, kommt Redner auf die neuern Angriffe anf die Paracentese des Trommelfells zu sprechen, bei deren Begrïndung der theoretisehen Speculation ein zu weit gehender Spielraum zugestanden worden ist.

Er giebt ein deutliches Bild der Stellang, welche die Paracentese seit ihrer wissenschaftlichen Begrlindung und Einführung in die Praxis dureh Schwartze vor nunmehr fast 
30 Jahren heute einnimmt, und führt die heute allgemein anerkannten Indicationen an, sich dabei eng an Schwartze anschliessend.

Dic früher bereits widerlegten Anfeindungen übergehend, wendet er sich zu einer kritischen Beleuehtung der von Piffl in seiner Arbeit "Acute Mittelohrentzündung und ihre Behandlung" vorgebrachten Angriffe gegen den Trommelfellschnitt.

Zunächst berührt er einige Punkte des theoretischen Theiles der Piffl'schen Arbeit, welche ihm für die Auffassung Piffl's nach der Seite der Therapie der acuten Otitiden hin von grundlegender Bedeutung zu sein scheinen.

Hierbei unterzieht er zunächst den Vorschlag Piffl's, anstatt der früheren Eintheilung der acuten Mittelohrentzündungen in acuten Catarrh und acute Eiterung, die Otitiden nach "aetiologischen" Gesichtspunkten in primäre und secundäre einzutheilen, einer abfälligen Kritik und betont, dass die von Piffl befürwortete Eintheilung niemals den Anspruch erheben könne, eine „ätiologische" genannt zu werden. Weiterhin kommt er eingehend auf diejenige Anschaung Z Zu fal-Piffl's zu sprechen, welche ihm die theoretische Grundlage der von jenen befürworteten Therapie zu sein scheint; die Anschauung, dass die acuten Otitiden normal einen cyklischen oder typischen Verlauf haben sollen, Beginn mit Schüttelfrost und kritischer oder lytischer Abfall der Temperatur am 7. oder 8. Tage.

Redner betont, weshalb er auf Grund seiner Erfahrungen in der Sehwartze'schen Klinik sich dieser Auffassung nicht anzuschliessen vermag.

Was die speciellen therapeutischen Vorschläge Piffl's anbetrifft, so empfiehlt letzterer eine Therapie, welche es ermöglichen soll, in der Regel die Paracentese bei der Behandlung der acuten Otitiden zu umgehen. Dieses Ziel bält er für erstrebenswerth, weil die Fälle ohne Perforation des Trommelfells im Allgemeinen rascher und günstiger verlaufen sollen und weil bei ihnen nicht die Gefahr der Secundärinfection und Cholesteatombildung vorliege. Er glaubt, durch heisse in essigsaurer Thonerde getränkte Wattecompressen anf $\mathrm{Ohr}$ und Warzenfortsatz in Verbindung mit innerlicher Darreichung von Natrium salicylicum dieses Ziel zu erreichen, sich ron dieser Therapie nicht nur einen prompten schmerzstillenden Effect, sondern auch eine gute Resorptionswirkung auf selbst eitriges Exsudat in der Paukenhöhle versprechend. Die Paracentese will er im Allgemeinen nur dann 
angewandt wissen, wenn am 7. oder 8. Tage Fieber und Sehmerz nicht aufgehört haben.

Redner entkräftet alle von $P$ iffl vorgebrachten Einwände gegen die Paracentese, insbesondere auch den, "dass dieselbe den Eintritt lebensgefährlicher Complicationen nicht zu verhüten vermöge". Er zeigt, dass uns die P iffl'sche Arbeit eigentlieh niehts Neues bringt, dass uns Piffl den Beweis schuldig geblieben ist für seine Behauptung, dass bei seiner Behandlungsart sich selbst ein eitriges Exsudat in der Paukenhöhle resorbiren könne, und dass gewisse in seiner Indicationsstellung sich findende Widersprüche nur dadurch ihre Erklärung finden, dass er die bewährte Eintheilung der acuten Otitiden in acuten Catarrh und acute Eiterung verlassen hat. Desgleichen widerlegt Redner den zustimmenden Siebenmann'schen Standpunkt.

Zum Schlusse hebt Redner hervor, dass es gewiss gut ist, Behandlungsmethoden, welche längst ein gesicherter Besitzstand der Therapie. zu sein scheinen mit dem Maassstabe, den ein grösseres Beobachtungsmaterial an die Hand gegeben, von Zeit zu Zeit nachzuprüfen; bedenklich sei es aber, ein Thema, wie das einschlägige, vor ein Forum von practischen Aerzten zu bringen, welche demselben kritiklos gegenüberstehen und dadurch irre werden könnten an ihren eigenen bisherigen günstigen Erfahrungen mit der Paracentese. (Autoreferat.)

\section{Discussion.}

Piffl (Prag) verwabrt sich gegen den Vorwurf, dass er unrecht gehandelt habe, die vorliegende Frage vor dem Forum von praktischen Aerzten zu verhandeln. Da man an der Zaufal schen Klinik zu der Ueberzeugung gekommen sei, dass die eingeschlagene Methode die richtige sei und $\mathrm{Zaufal}$ schon seit Jahren diese seine Methode in der Klinik gelehrt habe, so sei er wohl dazu berechtigt gewesen, den Gegenstand auch vor Aerzten, die zum grossen Theile Z a u fal'sche Schïler seien, zu besprechen. Dass Grunert den typisehen Verlanf der Mittelohrentzündung nieht beobachtet hat, liegt vielleicht daran, dass er den Verlauf einer solchen Entzündung immer durch die Paracentese unterbrochen hat. Uebrigens branche der Krankheitsverlauf nicht in jedem Falle 7 Tage zu dauern, sondern könne auch kürzer sein. Bei vorhandener Perforation könne durch Schnäuzen leichter Secret in das Mittelohr gelangen. Piffl hat die Beobachtung gemacht, dass Wärme besser vertragen wird 
als Kälte. Der ganze Artikel sei nieht etwa ein Kampf gegen die Paracentese uberhaupt, er sei nur gerichtet gegen die zu häufige Ausführung in den Fällen, wo die Erkrankung durch die ubrige Behandlung zurlickgehe. In Trier bätten sich verschiedene Ohrenärzte auf den Standpunkt Piffl's gestellt. Die Meinung, dass Complicationen durch Vornahme der Paracentese verhindert werden könnten, müsse dadurch erschtittert werden, dass, trotzdem manchmal in den ersten Stadien Perforation eintritt, Complicationen hinzukommen. Die Hauptsache sei doeh immer die Art der Infection. Alle, die gegen die Behandlungsmethode Z a ufal's sprächen, hätten dieselbe nicht nachgeprüft; Piffl hält deshalb die Einwände nicht für stichhaltig und glaubt die von ihm vertretene Ansicht noch aufrecht erbalten zu müssen.

Zaufal (Prag) will auf zwei Einwände erwidern: Er sei nicht auf Grund theoretischer Speculationen und bakteriologiseher Untersuchungen zu seiner Ansicht gekommen, sondern als Praktiker. Er sei ein grosser Anbäger der Paracentese gewesen wie alle Anderen, nur Schritt für Schritt sei er davon abgekommen. In seiner Privatpraxis sei er häufig Leuten begegnet, die die Vornahme der Paracentese nicht gestatteten. Nach 7-8 Tagen sei dann plötzlich ohne Paracentese ein Umschwung eingetreten. Das habe ihn veranlasst, dann öfters abzuwarten, und immer mit demselben guten Erfolge. Auf Grund der dann wieder vorgenommenen Paracentese habe er bei bakteriologischen Untersuchungen wieder gesehen, dass zu den rorbandenen Bakterien andere hinzutraten. Waren anfänglich nur Pneumokokken vorhanden, so traten später Streptokokken hinzu und umgekehrt. Auch wurde die Otorrhoe nach der Paracentese wieder viel stärker, als sie in den Fällen mit Spontandurchbruch war. Auf Umsehläge mit essigsaurer Thonerde seien auch Fälle mit Mastoiditis zurïekgegangen, ebenso wie bei paracentesirten Fällen. Durch diese Erfahrungen sei er zur Zurtickhaltung gekommen, also nicht auf theoretische Grïnde hin, sondern auf Grund praktischer Erfahrungen. Die Statistik hewiese nichts, man könne nicht behaupten, dass die Körner'sehen Fälle ohne künstliche Perforation nicht ebenso schnell geheilt wären.

Berthold (Königsberg) ist Anhänger der Paracentese. El fübrt Fälle an, wo er die Paracentese für unerlässlich hält. Die Sackträger (im Hafen von Königsberg) erkälteten sich bei ihrer sehweren Arbeit leicht; sie kämen dann oft mit schweren Erscheinungen einer Mittelohrentzündung in die Klinik, unter hef- 
tigen Sohmerzen. Nach der sofort vorgenommenen Paracentese heilte die Otitis schnell ab. Bei traumatischer Otitis in Folge Verschluckens von Wasser beim Baden oder beim Ausspritzen der Nase mache er sofort die Paracentese und habe gute Erfahrungen damit gemacht. Sofort Erleichterung und Heilung.

M iiller (Karlsbad) hat eine sehr sohwere Epidemie beobachtet, das typische Bild schwerer Influenzaotitiden mit hohem Fieber und starken Allgemeinerscheinungen. Wurde die Paracentese in den ersten 24 Stunden gemacht, so war der Erfolg gut. Bei anderen, die später in Behandlung kamen, trat die Heilung später ein. Andere, bei denen schon Spontanperforation eingetreten war, heilten sehr langsam.

Gomperz (Wien) hält die Methode Zau fal's für die leichten Formen der Mittelohrentzündung für gut. Sind Fieber, starke Schmerzen, Pulsbeschleunigung $u$. s. w. vorhanden, muss man bei der Paracentese bleiben, und zwar bei der frühzeitigen. Er habe früher mehr abgewartet und hat dann später erst mehr frühzeitig paracentesirt. Er hat von dem Zuwarten in den erwähnten Fällen nur Sehlechtes gesehen, von der frühzeitigen Paracentese nur Gutes.

Barth (Leipzig): Die schlechten Erfahrungen mit der Eisbehandlung haben sehr verschiedene Ursachen. Manehe Patienten sträuben sich gegen die Behandlung, weil sie dieselbe nicht kennen und wenden sie deshalb meistens schlecht an. Die Anwendungsweise sei aber von der grössten Wiehtigkeit. Der Eisbeutel müsse eingewickelt werden und nirgends frei auf der Haut liegen, da es sonst zu Gangrän kommen könne. Ferner müsse darauf geachtet werden, dass der Eisbeutel, wenn er gebraucht werde, permanent liegen bleibe. Wenn das nicht der Fall sei, komme es leicht zu unangenehmen Empfindungen. Man müsse selber öfter controliren, ob diesen Vorsehriften genügt werde, anf das Wartepersonal und den Kranken könne man sich nicht verlassen. Bei der Daueranwendung des Eisbeutels werde man anch Freude an dieser Behandlungsmethode haben.

Bezold (München) ist nicht zurückhaltend mit der Paracentese. Eiter in der Paukenhöhle werde allerdings aneh so resorbirt, wie die Sectionen an Masernleichen bewiesen haben. Er glaubt, dass die Eiterung im Mittelohr eine Bagatelle sei gegen die in den Zellen. Für lebensrettend halte er die Paracentese nicht, halte sie aber für dringend indicirt bei alten Leuten, wo das Gewebe nicht so resorptionsfähig sei, und dann in solehen 
Fällen, wo bei vorher intactem Labyrinth die obere Tongrenze anfängt eingeschränkt zu werden.

Grunert (Halle) wendet sich gegen den Vorwurf, dass er nie einen Fall von Mittelohrentzündung, ohne dass derselbe durch einen künstlichen Eingriff gestört wurde, beobachtet habe. Es gehe aus den statistischen Zahlen des Poliklinischen Journals der $\mathrm{Hall}$ e'sehen Ohrenklinik hervor, dass die Paracentese in der Hälfte der Fälle überhaupt nicht in Frage gekommen sei. Diese Fälle seien doch auch ohne Paracentese beobachtet. In der Halle'schen Klinik stehe man nicht auf dem Standpunkte, sofort zu paracentesiren, sondern man warte selbst in heftigeren Fällen bei antiphlogistischen Mitteln erst 24 Stunden $a b$, and mache erst die Paracentese, wenn dabei keine Besserung eintrete. Für Fälle, wo man sicher Eiter erwarten könne, gelte der Grundsatz: Ubi pus, ibi evacua.

3. Kümmel (Breslau): Fraisen zur Eröffnung des Warzenfortsatzes, mit Demonstration.

Die angegebene Fraise hat den Vortheil, dass sich dieselbesterilisiren lässt. $\mathrm{K} u ̈ \mathrm{~m} \mathrm{~m}$ el meint, dass die Fraise in einzelnen Fällen, wenn sie sonst gut brauehbar sei, dem Meissel vorzuziehen sei. Er glaubt, dass es durch die Meisselsehläge doch zu einer Meningitis kommen könne, wenn nämlich schon Eiter im Labyrinth sei.

Schwartze (Halle) wendet sich gegen die Unterstellung, dass er nicht damit einverstanden sein möchte, dass durch den Meissel hier und da eine Meningitis hervorgerufen werden könne. Er zweifelt nicht, dass ein Hirnabscess, der nahe am Durchbruch ist, durch die Erschütterung perforiren kőnne, derselbe würde dann aber auch ohnedem zum Durchbruch gekommen sein. Es komme bei der Anwendung des Meissels viel auf die Art des Gebrauches an. Man dirfe denselben nicht wie ein Zimmermann, sondern wie ein Bildhauer gebrauchen. Besonders bei sehr sklerotisehem Knochen könne es wohl auch einmal durch die Erschïtterung zur Propagation einer Labyrintheiterung kommen, dieselbe würde aber auch spontan eingetreten sein. Wenn $\mathrm{K} u ̈ \mathrm{~mm}$ el sage, Macew en perhorreseire neuerdings den Meissel, so sage er (Sehwartze), er perhorreseire die Fraise, weil ihm die Führung derselben zu unsicher sei und er beim Gebrauch derFraise die Wunde nicht so gut ubersehen kỏnne, als beim Meissel. Bei dem Gebrauche des letzteren erscheine ihm Sinus und Dura weniger gefährdet als bei der Fraise.

4. Katz (Berlin): Demonstration mikroskopiseher Präparate (betreffend das membranöse Labyrinth). 
Cholesteatombildnng, Erkrankungen des Hammeramboss-Gelenkes, rareficirende Ostitis und stereo. skopisebe Bilder.

Katz bespricht zunächst die Methode, nach welcher die vorliegenden mikroskopischen Präparate des membranösen Laby rinths conservirt resp. hergestellt sind. Für das wichtigste und relativ sicherste Mittel, die äusserst zarten, leieht veränderliehen nervösen Epithelgebilde des Corti'schen Organs, sowie der Macula aoustica und Crista acustica zu conserviren, hält $\mathrm{Katz}$ die Osmiumsäure (1/3-1/2 Proc.). Das vorsichtig geöffnete Labyrinth muss möglichst frisch in eine solche Lösung für $2-3$ Stunden gebracht werden, dann weiter in eine ca. 1/2 procentige ChromEssigsäurelösung und dann zur Entkalkung je nach der Dieke des Knochens in 5-10 procentige Salpetersäure- resp. Salzsäurelösung. Katz ist, auf Grund eigener Erfahrung der Ansicht, dass an menschlieben Felsenbeinen, die nur in Muller'scher Flüssigkeit oder selbst in der entschieden vorzuziehenden F or mol Mủller-Lösung gehärtet sind, das Neuroepithel an der Papilla basilaris und an der Macula und Crista nicht tadellos erhalten werden kann. Fehlsehlüsse in pathologisch-anatomiseher Hinsicht sind dabei of nieht zu vermeiden und kommen thatsächlich häufig in der Literatur vor.

1. Das erste Präparat, welches Katz demonstrirt, betrifft ein Corti'sches Organ einer Katze.

Hier weist Katz auf die merkwürdige Verbindung zwischen äusseren Corti'schen Zellen und Deiters'schen Zellen hin. Im Arehiv für mikroskopisehe Anatomie bat nenerdings (Bd. LIX) der japanisehe College Ichita Kishi in seinen Schlusssätzen Folgendes behauptet:

"Die Endfasern des Nervus cochleae, welche zu den Haarzellen treten, gehen je eine in ein bisher noch von $\mathrm{Nie}$ mand beschriebenes, an dem unteren Ende der Haarzellen befindliches Gebilde von kelchförmiger Gestalt über, welches ich als einen Teil der Haarzellen betrachte."

Zunächst urgirt Katz, dass das "kelehförmige" Gebilde von $\mathrm{K}$ is hi bereits von ihm ( $\mathrm{K}$ a tz) im Jahre 1888 in der Monatsschrift für Ohrenheilkunde mit Abbildungen besehrieben und "Zangenbecher" genannt worden ist. Der Titel seiner damaligen Arbeit lautete: "Beitrag zurFrage über die Verbindung der Corti'schen und Deiterssehen Zellen". Katz hat damals an der Hand von Zupfpräparaten, besonders von Fledermäusen, den Nachweis geftihrt, 
dass das von ihm gesehene fibrilläre, mit der Stuitzfaser zusammenhängende "zangenförmige Gebilde" nicht za der Stäbchenzelle (Corti'sche Zelle), wie dies $\mathrm{K}$ is $\mathrm{i}$ behauptet, sondern zu del" Deiters'schen Zelle gehôrt. In dem "Zangenbecher" ruht das untere Ende der Stäbchenzelle, wie „ein Ei in ein em Eibecher". - Was die Endigung der änsseren radiären Fasern des Nervus cochleae betrifft, so hat Katz auf der Versammlung: der Deutschen otologisehen Gesellschaft in Dresden 1897 unter Vorzeigung von Präparaten sich dahin ausgesprochen, dass die Nerven-Fasern an dem unteren Ende der Corti'seben (Stäbehen) Zellen sich filzartig auflösen resp. das untere Ende fibrillär ein-

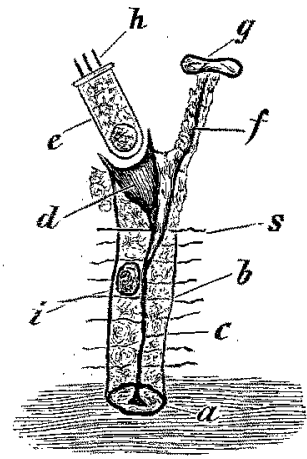

Fig. 1.

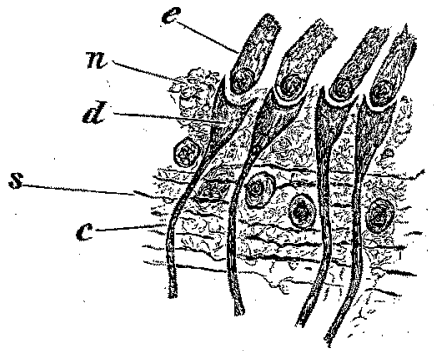

Fig. 2.

Verbindung der Corti'sehen und Deiters'sehen Zellen. Schema.

$e$ äussere Corti'sehe Zelle (äussere Stäbhenzelle), $d$ Zangenbecher, $s$ spirale Fasern, $c$ Stitzfaser inmerhalb der Deiters'sehen Zelle, $i$ Kern der Deiters'sehen Zelle, $p$ polygonale Basis der Deiters'sohen Zelle, $g$ Phalange, $f$ Phalangenfortsatz, $h$ Haare (Stäbchen), $n$ zu einem fibrillären Knäuel aufgelöster Nerv. cochleae.

hüllen (sie hängen nicht mit dem Zangenbecher zusammen).

Die äusseren spiralen Fasern, die das untere Ende der Deiters'schen Zellen berühren, hält Katz nicht für nervöse Fasern; sondern für Stützapparate für das ganze Corti'sche Organ. Der Nervus cochleae endigt nach $\mathrm{K}$ at $z$ analog dem Nervus vestibuli durch fibrilläre Einhüllung der Haarzellen an der Crista und Macula.

2. Ein ferneres Präparat betrifft die normale Lage der Membrana tectoria. Dieselbe liegt dem Corti'sehen Organ auf, resp. berïhrt die Stäbchen der Corti'sehen Zellen. Das Umschlagen der Membrana tectoria ist stets ein Artefact. Die Membran ist ferner nicht an der gegenüberliegenden Wand des Duetus coch- 
learis fixirt, wie das einige Autoren, u. A. College Barth, annimmt.

3. Ein anderes Präparat pathologischer Natur betrifft das von $\mathrm{Kir} 0 \mathrm{hner}$ angegebene Eindringen von Cholesteatomelementen in die Havers'schen Canäle. Katz hat schon früher darauf hingewiesen, dass er diesen Befund von Kirchner niemals in seinen Präparaten bestätigt gefunden hat. Auch dies neue vorliegende Präparat zeigt kein derartiges Eindringen von Epidermiszellen in die Havers'sehen Canäle, dagegen finden sich hier in der Umgebung des. Cholesteatoms in den grösseren und kleineren Markräumen reichliche Fettzellen, welche gequollenen Epidermiszellen sehr ähnlich sehen.!

4. Querschnitt duroh das Hammer-Ambossgelenk eines ca. 1 Jahr alten, an chronischer Otorrhoe leidenden Kindes.

Der entzündete Knochen dieser beiden Gehörknöchelchen ist von auffallend grossen und zahlreichen Markräumen durchsetzt, in welchen sich ein sehr zellenreiches, junges Bindegewebe, sowie zahlreiche erweiterte Gefässe nachweisen lassen; den Process bezeichnet Katz als rareficirende Ostitis. Ein derartiger Entzündungsprocess in der Paukenhöhle führt bei bestehender krankhafter Disposition theils zum Sehwund des Knochens (rareficirende Ostitis), theils an anderen Stellen zur osteoplastischen Auflagerung (Hyperostose; Exostose, Sklerose u. s. w.).

Von der Caries ist diese rareficirende Ostitis histologisch allerdings nur quantitativ unterschieden; ein Uebergang einer rarefioirenden Ostitis in eine Caries ist nach $\mathrm{Katz}$ unter prädisponirenden Umständen (Tuberculose, Scrophulose, Syphilis u. s. w.) leicht möglich. Im vorliegenden Falle ist der Process wesentlich auf das Innere der beiden Gehörknöehelehen localisirt. - Die Contouren der Gehörknöchelchen haben sich nicht verandert. Solche rareficirende Processe sind, wie $\mathrm{Katz}$ angiebt, n a eb und bei Otitis media chronica nicht selten. Sie können aber auch zur relativen Ausbeilung gelangen, wie aus einem gleichzeitig vorgelegten Präparate hervorgeht. In letzterem sind neben Bindegewebsneubildung in der Pauke die Gehörknöchelchen von schwammartigem (osteoporotischem) Aussehen, während an anderen Stellen der Pauke sich Osteophytenbildung (Hyperostose u. s. w.) constatiren lassen, Erscheinungen, wie man sie auch bei sogenannter Otosklerose ofter findet and welche intra vitam nach Katz sicherlich die gleichen klinischen Symptome zu Stande bringen. Nach Katz's Ansicht kann demnach bej bestehender 
Disposition des Knochensystems aus jeder Otitis media, sei es, dass sie schleichend und latent oder acut und manifest verläuft, eine sogenannte Sklerosis entstehen, die eventuell zur Ankylosis des Steigbügels fübrt.

5. Endlich demonstrirt $\mathrm{Katz}$ stereoskopische Ansichten des gesammten Labyrinths und der Pankenhöhle. Die Präparate sind nach der von $\mathrm{Katz}$ früher beschriebenen Methode dargestellt (dieses Arehiv Bd. XXXV). Autoreferat.

Dritte Sitzung, gemeinsam mit der rhino-laryngologischen Abtheilung: 23. September, Nachmittags 2 Uhr.

Vorsitzender: Heymann (Berlin).

1. Barth (Leipzig): U eber Täusehungen des Hörens in Bezug auf Tonhöhe und Klangfarbe.

Durch experimentelle Versuche hat Barth die Richtigkeit der Helmboltz'schen Annahme nachgewiesen, dass durch Verändernngen in der Zusammensetzung der Obertőne jeder Ton in seiner Klangwirkung verändert wird, so dass er böher oder tiefer erscheint. Selbst musikalisohe Personen vermögen vielfach diese Veränderung der Tonhöhe nicht richtig zu erkennen und anstatt der Veränderung der Klangfarbe vermuthen sie oft eine veränderte Stellung des Tones in der Tonleiter.

Die Veränderung der Klangfarbe kommt theilweise schon bei der Entstehung des Tones zu Stande, theils auch bei der Fortleitung im schallleitenden Apparate.

\section{$D$ iscussion:}

Gutzmann (Berlin): Die Versuche von Barth und von Spiess unterscheiden sich dadurch, dass sich bei dem letzteren ein Sinken des Tones direct nachweisen lässt.

Berthold (Königsberg): Die Ursache der Diplakusis liegt nicht im schallleitenden Apparate, sondern im Corti'soben Organe.

2. Anton (Prag): Demonstration uber die Tuben, Paukenhöhlentonsille and congenitale Deformitäten der Nasensebeidewand.

a) Bei 56 Kinderleichen (im Alter von 6 Stunden bis 40 Tagen) fand Anton 9 mal Deformitäten am Septum, und zwar meist Leistenbildungen, und $1 \mathrm{mal}$ eine Deviation. Meist verlief die Leiste dicht über dem Nasenboden und wurde nach hinten zu flacher. Oft war Schiefstand des Septums damit verbunden. Die Leiste kommt entweder durch das seitliche Aus- 
weichen der Cartilago quadrangularis zu Stande oder dadurch, dass Knochen und Knorpel sich in einem Winkel berubren.

b) Anton zeigt einige Präparate mit lymplatischem Gewebe in der Pars ossea tubae und in den angrenzenden Partieen der Paukenböhle. Dasselbe liegt meist in Schleimhautfalten, oft in Form von zapfenförmigen, follikelartigen Herden und Infiltraten.

\section{Discussion:}

Heymann (Berlin) hat am Septum von Embryonen öfter Abweichungen beobachtet.

Habermann (Graz): Die zapfenförmigen Gebilde kommen auch an der Innenseite des Trommelfells vor. Habermann hat 3 solche Fälle beobachtet.

3. Heymann (Berlin): Zur Anatomie und Pathologie der Nebenhöhlen.

4. Hoffmann (Dresden): a) Ueber Kiefercysten; b) Ein Fall von Wangenabscess nach Kieferböhleneiterung.

Discussion: Biehl, Imbofer.

5. Müller (Karlsbad): Behandlung der Catarrhe der oberen Luftwege mittelst Spraydouchen-Hochdrueksystem.

6. Löwe (Berlin): Ueber die Ausräumung der Nase vom Munde her.

7. Löwy (Karlsbad): a) Ein Kieferhöhlenempyem bei Diabetes; b) Dysmenorrhöe geheilt nach nasaler Therapie; e) Adrenalin in der rhino-otologisohen Praxis; d) Zur Inhalationstherapie.

Discussion: Urbantschitsch, Katz, Berthold, Hoffmann.

Vierte Sitzung: Nachmittags 5 Uhr. Vorsitzender: Berthold (Königsberg).

1. Piffl (Prag): Zur Freilegung des Bulbus venae jugularis. (Mit Demonstration.)

Für die Fälle, bei denen in Folge ungünstiger anatomischer Verhältnisse der Bulbus nach der Methode von Grunert nicht zu erreichen ist, ferner bei Verdacht auf isolirte Bulbusthrombose und schliesslich zur Beseitigung erkrankten Knochens am Boden oder an der Innenwand der Paukenhöhle empfieblt Piffl die Blosslegung des Bulbus durch Abtragung der vorderen und 
unteren Gehörgangswand, des Paukenhöhlenbodens, wenn nöthig eines Theiles der inneren Wand der Paukenhöhle und des Daches des Recessus jugularis, in ungünstigen Fällen anch des Processus styloid. sammt Scheide und dem Rest der hinteren Gehörgangswand bis zum. Canalis fallop. - An dem so freigelegten Bulbus lässt sich dann Punction, Ausräumung, Tamponade u. s. w. vornebmen. - Piffl demonstrirt ein Präparat, an welchem diese Operation ausgefuhrt ist, und 2 nene Zangen, die den vorliegenden Knoehenverhältnissen angepasst sind. (Autoreferat.)

2. Grunert (Halle a. S.): Beitrag zur operativen Be handlung der infectiösen Thrombose im Bulbus venae jugularis. (Ist ausfuhrlich in diesem Arehiv Bd. LVII erschienen.)

\section{Discussion:}

Kümmel (Breslau) hat auch in 2 Fällen die Bulbuseröffnung vorgenommen, von denen einer geheilt, einer letal verlaufen ist. Der Bulbus ist manchmal eigenthtimlich geformt, so dass es schwierig ist, ihn vollständig zu eröffnen. Auch die Arteria stylomastoidea sei manchmal im Wege. Kü mmel empfiehlt statt der Sptilung durch den Bulbus, Jodoforminjectionen in denselben zu machen, $\mathfrak{u m}$ den "Dom" damit zu desinficiren. Auch eine Facialisverletzung lasse sich manchmal nicht vermeiden.

Piffl (Prag): Die Blutung war bei seinen Fallen keine bedentende. Da die hintere Wand nicht entfernt werde, komme man der Arteria stylomastoidea nicht nahe. Die Facialisverletzung müsse man mit in den Kauf nehmen.

Alexander (Wien): Facialisverletzungen haben nichts za sagen, wohl aber Verletzungen des Vagus, der dem Bulbus nahe liege. Er glaubt, dass diese Verletzung unvermeidlich sei.

Grunert hat nie Symptome von Vagusverletzungen bei Bulbusoperationen gesehen und weist die aus theoretischer Voreingenommenheit and nicht aus kliniseher Erfahrung hervorgehenden Bedenken Alexander's zurück.

Kümmel glaubt auch nicht, dass eine Vagusverletzung etwas zu bedeuten habe.

Sehwartze weist auf die gesehiitzte Lage des Vagus hin; eine Collision mit demselben könne nur bei Jugularisunterbindung vorkommen, andere Erscheinungen als Athembeschleunigung habe er als Folge davon nicht gesehen. 
3. Gomperz (Wien): Demonstration neuer küntlicher Trommelfelle.

In einzelnen Fallen von Trommelfelldefecten wird keins der bisher construirten'künstlichen Trommelfelle vertragen, und zwar da, wo die Paukenhöhlenschleimbaut als solche sich erhalten hat. Ref. hat sich bemüht, auch für diese Fälle Trommelfelle zu construiren. Nach vergeblichen Versuchen mit Celloidinplatten u. s. w. spritzte er eine Lösung von sterilisirtem Vaselin-Paraffin in den Gehörgang ein und erzielte damit recht gute Resultate. Die Masse wurde absolut reizlos vertragen, und er konnte sie monatelang liegen lassen, auch das functionelle Resultat war gut. Auch Plättchen von chemisch reinem Silber wurden ohne die geringste Reizung vertragen. Dazu liess er sich ganz düne Plättchen von $4 \mu$ Dicke herstellen. Dieselben lassen sich leicht sterilisiren, und werden dann, nachdem der Gehörgang mit Alkohol und Aether ausgewischt ist, zusammengekrüllt in die richtige Lage gebracht. Am besten haben sich ihm die Blättehen aus Schanmsilber bewäbrt.

\section{Discussion:}

Katz (Berlin) meint, dass sich auch diese Blättchen leicht verschieben könnten, und dann ihren $Z$ week verfehlen.

Berthold (Königsberg) weist darauf hin, dass er früher schon Hühnereihäutchen für den Zweck empfohlen habe, und dass diese denselben $\mathrm{Z}$ week erfüllen.

Gomperz hält dieselben deshalb nicht für brauchbar, weil sie sich nicht sterilisiren lassen.

Schwartze weist darauf hin, dass Lucae bereits in Hamburg mitgetheilt habe, dass die Wirkung des kunstlichen Trommelfells unabhängig von der Berührung mit dem Steigbügel sein könne."

4. Breitung (Coburg): a) Ueber die Verwendung elektromagnetisch bewegter Stimmgabeln in der otologischen Praxis (Demonstration); b) Automatisohe Regulirung des Kolbenhubes bei der elektromotorischen Luftpumpe (Demonstration).

Fünfte Sitzung: Mittwoch, den 24. September, Nachmittags.

Vorsitzender: Barth (Leipzig).

1. Katz (Berlin): Anatomische Demonstrationen des $G$ ehörorganes mit Projectionen. 
1) Durchschnitt durch die Schnecke eines jungen Löwen; der Modiolus ist besonders kräftig gebaut und gleicht darin demjenigen der Katze.

2) Durehschnitt durch das ganze Labyrinth und den im ovalen Fenster befindlichen Steigbügel. Besonders deutlich sind die beiden Säckehen und der Aquaeductus vestibuli.

3) Querschnitt durch die Tuba Eustachii vom Mensehen im oberen Theil; topographisches Verhältniss derselben zur Carotis interna.

4) Durchschnitt durch das Labyrinth einer Katze; zu sehen ist besonders der Sacculus, der Ductus reuniens Hensenii und der Anfang des Duct. cochlearis resp. der Membr. basilaris.

5) Schnitt durch das runde Fenster; die Lamina spiralis ossea et membranacea sind durch einen tangentialen Schnitt 2 mal in derselben Ebene getroffen.

6) Querschnitt dureh den Steigbügel und den Musculus stapedius nebst Sehne.

7) Horizontaler Querschnitt durch den Atticus des Menschen. $\mathrm{Zu}$ bemerken ist der horizontale Theil des Nervus facialis und das Knie desselben.

8) Querschnitt durch das ovale Fenster und den ankylotischen Steigbügel bei Otosklerosis.

9) Frontaler Schnitt durch Paukenhöhle und Labyrinth bei einem Fall von Otosklerosis. Der ostitische stark vascularisirte Herd sitzt im Schnitt ganz in der Nähe des runden Fensters und ist deutlich begrenzt.

10) Schnittdureh die Schnecke eines schwerhörigen Menschen (Otosklerose). Die Scala tympani ist derartig erweitert, dass die Lam. spir. ossea et membranacea in allen Windungen stark nach oben ausgebogen erscheint, wäbrend die Membran des runden Fensters in anderen Schnitten desselben Präparates in entgegengesetzter Richtung, also nach aussen convex ist. Es handelt sich nach $\mathrm{Katz}$ hier um einen perilymphatischen Ueberdruck während des Lebens in der Scala tympani der Sehnecke.

11) Querschnitt durch die Sehnecke eines erwachsenen Taubstummen. Zwischen den beiden Blättern der Lam. spir. ossea ist der Nervus cochleae in den beiden unteren Windungen hochgradig atrophisch, Ganglienzellen fehlen fast ganz.

12) Längsschnitt durch das Trommelfell bei Otit. med. pur. mit feiner Perforation. Man sieht deutlich mässige Zitzen- 
bildung und Umsehlagen der Epidermis des Trommelfelles in die feine Perforationsöffnung hinein. Die Catisschicht des Trommelfells sehr verdickt, ebenso die Schleimhantschicht der Paukenhöhle.

13) Atrophie des Nerv. cochleae in einem Querschnitt durch die Schnecke eines Taubstummen; die Lam. spir. ossea sehr verdünnt.

14) Kleines Cholesteatom im Atticus. Quersehnitt durch die Paukenhöhle.

15) Hypertrophie der Schleimhaut der Paukenhöhle bei Otitis med. acut., an der Membran des runden Fensters sitzt eine zapfenartige Schleimhautwucherung, welche die Membran nach aussen zieht.

16) Frontaler Schnitt durch Paukenhöhle und Labyrinth. Der Hammer ist in seiner Längstichtung getroffen. Prussakscher Raum sichtbar. - Es handelt sich um einen Fall von Otosklerosis, in der Wand des Promontoriums unterhalb des Nervus facialis ist ein circumseripter ostitischer Herd deutlich sichtbar; innerhalb des Herdes sehr zahlreiche, neugebildete weite Blutgefässe.

17) Sackförmiges, grosses Cholesteatom der Paukenhöhle. Die Matrix des Sackes beginnt innerhalb des äusseren Gehörgangs und überzieht bei fast vollständig fehlendem Trommelfell den Steigbügel sowie die stark gewulstete Schleimhaut der Paukenwände.

18) Starke Zerstörung der Schleimhaut der Pauke sowie cariöse Defecte der knöchernen Labyrinthkapsel bei Tuberculosis; fibrinöse Entzündung in den Scalen.

19) Grosses Cholesteatom innerhalb der Paukenhöhle; die knöchernen Wände sind theils hyperostotisch, besonders am Steigbügel, theils an anderen Stellen rareficirt (osteoporotisch).

20) Abgelaufene rareficirende Ostitis (Osteoporose) der Gehörknöehelehen und der benaehbarten Atticuswand. Die theilweise auch zur Hyperostose führende Knochenerkrankung erstreckt sich an einer Stelle bis tief in die knöcherne Labyrinthkapsel hinein. Im Atticus sonst dichtes Bindegewebe, als Zeichen früherer Entzündung. -

Der Vortragende betont, dass sich derartige Projectionen flur Lehrzwecke besonders eignen und das Studium dieses complieirten Abschnittes des Organismus nicht unwesentlich erleichtern. (Autoreferat.) 
74. Versamml. deutsch. Naturforscher u. Aerzte in Karlsbad (otol. Sect.). 125

2. Alexander(Wien): a) Anatomisch-physiologische Untersuchungen an Thieren mit angeborenen Labyrinthanomalien (mit Demonstration lebender Thiere und histologiseher Präparate).

Die Untersuchungen erstreckten sich auf taube Katzen und Tanzmäuse, und zwar auf das physiologische Verhalten der Thiere und den anatomischen Bau ihrer Gehörorgane. Die Thiere beanspruchen unser Interesse in zweifacher Richtung: zunächst hinsichtlich des zoologischen Befundes und seiner Einzelheiten, dann auch, weil sie ein Beispiel für die degenerative Schwerhörigkeit oder Tanbheit darstellen, und die Befunde in Analogie mit den bei einer Form angeborener Taubheit des Menschen erhobenen Thatsachen gebracht werden können.

Alexander bespricht zunächst das physiologische Verhalten tauber Katzen. Die anatomische Untersuchung ergab:

1) Aufhebung der normalen Gestalt der Pars inferior labyrinthi, die häutigen Wände sind aneinander gelagert, zum Theil bindegewebig aneinander fixirt.

2) Destruction und Degeneration der Nervenendstellen der Pars inferior (Macula sacculi und Papilla basilaris cochleae).

3) Gänzlichen Pigmentmangel des unteren Labyrinthabschnittes.

4) Atrophie des Ganglion und Nervus cochleae. - Die Tanzmaus weist nicht bloss im Gehörorgan, sondern auch im Gleichgewichtsapparat Veränderungen anf. Ihre physiologische Untersuchung ergab:

1) Die Tanzmäuse reagiren auf keinerlei Schalleindrücke.

2) Sie besitzen ein mangelhaftes Vermögen, das Körpergleichgewicht zu erhalten.

3) Sie haben keinen Drehsehwindel.

4) Sie verhalten sich der galvanischen Durchströmung des Kopfes gegenüber wie normale Thiere.

Aus der anatomischen Untersuchung der Tanzmäuse resultiren folgende Thatsachen:

1) Destruction der Papilla basilaris cochleae mit verschiedengradigem Uebergreifen der Veränderungen auf die Gewehe der Umgebung. cochleae).

2) Hochgradige Atrophie des Ramus inferior n. octavi (Nerv.

3) Hochgradige Atrophie des Ganglion spirale. 
4) Destruction der Macula sacculi.

5) Mittelgradige Verdünnung der Aeste und Wurzeln des Ramus superior und medius n. octavi.

6) Die Bogengänge und Ampullen sind vollkommen normal geformt.

b) Zur Histologie der Hörnervenatrophie des Menschen (mit Demonstration histologischer Präparate).

Dem Vortrag liegt ein im Leben beobachteter Fall von progressiver Schwerhörigkeit zu Grunde, bei welohem die histologische Untersuchung der beiden Gehörorgane und des Hirnstammes eine beiderseitige hochgradige Atrophie des Schneckennerven, des Spiralganglion und des Corti'sohen Organs zu Tage gefördert hat. Die übrigen Theile des inneren Ohres, die mittlere und äussere Ohrsphäre erwiesen sich vollkommen intact, desgleichen der centrale Acusticus und die Acusticuskerne. (Autoreferat.)

\section{Discussion:}

Habermann (Graz) hat einen 70 jährigen, an Arteriosklerose leidenden Mann beobachtet, bei dem sich eine plötzliche hochgradige Zunahme der Schwerhörigkeit einstellte. Eine solche ist wabrscheinlich niemals rein nervös.

Katz (Berlin) räth im Ansebluss an die Demonstration von Alexander dringend zur Vorsicht bei der Beurtheilung von morphologisehen Veränderungen an der Papilla basilaris (Cort. Organ) und den maoulae und cristae. Selbst geringe Conservirungsfehler bewirken die mannigfaltigsten Veränderungen an den äusserst zarten Epithelgebilden.

Berthold (Königsberg) ist derselben Ansicht wie Habermann.

Barth (Leipzig) macht darauf aufmerksam, dass bei plötzlichem Eintritt hochgradiger Schwerhörigkeit auch centrale Ursachen eine Rolle spielen können. Redner führt einen entsprechenden Fall an.

Gomperz (Wien) kann Katz nicht Recht geben. Er hat anch bei guter Fixirung (Ueberosmiumsäure) oft Veränderungen constatiren können.

3. Buhe (Halle a. S.): Giebt es Anastomosen zwischen den Gefässbezirken des Mittelohres und des Labyrinths? (Ausführlich in diesem Archiv Bd. LVI abgedruckt.) 


\section{Discussion:}

Alexander (Wien): Wenn Braunstein und Buhe keine Anastomosen gesehen haben, so ist daraus nicht zu schliessen, dass thatsächlich keine bestehen. Denn Politzer habe solohe festgestellt. Die Berufung auf die Untersuchungen Eichler's hält Alexander nicht für beweiskräftig; Eichler starb bald nach Vollendung seiner Arbeit, hätte er länger gelebt, so hätte er vielleicht seine Anschauung geändert.

Katz (Berlin) hat niemals directe Anastomosen gesehen. $\mathrm{Er}$ hält das Vorkommen derselben sowohl nach der klinischen Erfahrung als auch vom entwicklungsgeschichtlichen Standpunkt aus für unwahrscheinlich.

Habermann (Graz) hat ebenfalls keine directen Gefässverbindungen feststellen können.

Alexander behauptet, wenn andere Untersucher keine Anastomosen gefunden hätten, so läge dies an der Methode. Die hierzu von ihm empfohlene Injectionsmethode hat er selbst, wie er auf Befragen Habermann's erklärt, für diesen Zweck nicht in Anwendung gebracht. Alexander behauptet, dass in der Politzer'schen Sammlung ein die Gefässverbindung beweisendes Präparat vorhanden sei, welches hier vorzulegen er nicht in der Lage sei.

$\mathrm{Bu}$ le weist darauf hin, dass er nicht bestreitet, dass in den Politzer'schen Osmiumsäurepräparaten ans chein end Anastomosen zwisehen den Gefässen des Mittelohres und des Labyrinthes vorhanden seien. Er selbst habe das zuerst bei Anwendung dieser Methode geglaubt. Dieselbe biete eben nicht genügend differenzirte Bilder und sei deshalb für den vorliegenden Zweok nicht beweiskräftig. Die von ihm ( $\mathrm{Buhe}$ ) eingeschlagene Methode liefere für die gegentheilige Ansicht einen dreitachen Beweis: erstens durehsetzen die rom Mittelohr in den Knochen eintretenden Gefässe selbst nie die Compacta vollständig, zweitens sähe man auch nirgends Bindegewebe, von dem die Blutgefässe ja überall begleitet wären, durch die mediale Sehicht der Compacta hindurchtreten und drittens gäben die Labyrinthgefässe, die man auf vielen seiner Präparate um die halbe Schneckenwindung verfolgen könne, nirgends auch nur andeutungsweise eine Anastomose nach aussen ab. Ausserdem stünde die von ihm vertretene Ansicht, wie erwähnt, mit den von Eichler gemachten Befunden im Einklange, dessen ausgezeichnete Arbeit A lexander selbst rühmend anerkannt habe. 
4. Biehl (Wien): Heben trockene, "bleibende" Lücken im Trommelfell in jedem Falle die Tauglichkeit zum Waffendienste auf?

Der Vortragende spricht sich in seinen Ausfibrungen gegen die derzeit noch bestehende, dem Titel gleichlautende Vorschrift für den österreichischen militärärztlichen Dienst aus. Er begrtindet seine Anschauung aus den "Ergebnissen der Sammelforschung tiber Krankheiten des Ohres im k. u. k. Heere in den Jahren 1897-1899". Diesen zufolge waren unter 10457 ohrenkranken Mannschaftspersonen 659, welche eine bleibende, trockene Liicke im Trommelfelle hatten. Von diesen sind 590 pro mille als "diensttauglich", 350,5 pro mille als " superarbitirt" und 259,5 pro mille auf "andere Art" abgegangen; gestorben Niemand. Weiter sprechen für die Taugliehkeit derartiger Leute die Erfahrungen in der deutschen Armee, in welcher seit dem Jabre 1882 die „bleibende Durchlöcherung des Trommelfelles" keine Ursache der Dienstunbrauchbarkeit mehr ist.

Als Behelf bei der Untersuchung empfiehlt er wärmstens das von Leutert angegebene Schema (Münchner med. Wochensehrift. Nr. 39, 40 u. 41. 1900). (Autoreferat.)

\section{Diseussion:}

Schwartze (Halle a. S.) ist der Ansicht, man solle Lente mit trockenen Perforationen vom Heeresdienst ausschliessen, einerlei, ob die Perforation auf Caries deutet oder nicht, da solche Leute mit trockener Perforation leicht einem Recidiv der Eiterung ansgesetzt sind und dadurch dem Staate zur Last fallen können.

Zaufal (Prag) ist ebenfalls dafür, alle Leute mit Perforationen auszuscheiden. Er hält dies für das Beste für die Kranken (aus Humanität) und für die Aerzte. Zum Mindesten müssten solche Leute zu besonderem Dienste eingestellt werden.

Alt (Wien) schliesst sich im Grossen und Ganzen der Ansicht von Schwartze und $\mathrm{Zaufal}$ an, schlägt aber vor, Leute mit trockener Perforation zunächst einzustellen und zu beobachten, und falls ein Schluss der Perforation möglich ist, für dienstfähig zu erklären.

Biehl (Wien): In der österreichischen Armee werden Leute mit trockener Perforation weder zu den Pionier- und Eisenbahntruppen, noch zur Artillerie genommen. 
5. Buhe (Halle a. S.): Functionelle Endresultate nach der Totalaufmeisselung des Mittelohres. (Ausführlich im LVI. Bd. dieses Arohirs.)

\section{Disoussion:}

Gomperz (Wien) weist auf seine diesbezüglichen Untersuchungen hin, die freilich an einem kleinen Material angestellt sind. Anlass zu den Untersuchungen gaben zwei Fälle, welche $6-7 \mathrm{~m}$ hörten. Bei diesen war der Steigbügel normal. Bei den schlechter Hörenden war der Steigbügel defect, entweder fehlte das Köpfchen oder die Schenkel. Gomperz hält das Intactsein des Steigbügels für wichtig für das Hörvermögen bei den Operirten.

Piffl (Prag): Bei Sectionen ist der Steigbügel wegen der Schwellung der Sehleimhaut oft sebwer zn finden. Piffl glaubt, dass bei dicker Epidermisauskleidung der Wundhöhle das Intactsein des Steigbügels sich schwer nachweisen lässt.

Sechste Sitzung: Donnerstag, den 24. September, Vormittags 9 Uhr.

Vorsitzender: Katz-Berlin.

1. Schwartze (Halle): Ueber Varietäten im Verlaufe des Facialis in ihrer Bedentung für die Mastoidoperation. (Der Vortrag ist in diesem Archiv ausführlich veröffentlicht.)

\section{Diseussion:}

Bezold (München): Die Regenerationsfähigkeit des Facialis sei eine grosse. Er habe gesehen, dass grosse Sequester aus der Facialisgegend ausgestossen wurden, ohne dass eine Lähmung zurückgeblieben sei. In einem Falle sei sogar der Facialiseanal in dem Sequester gelegen gewesen, der Faeialis musste also durchrissen sein, trotzdem sei die Facialislähmung geheilt. Auch bei Labyrinthsequestern habe er öfters Facialisparalysen verfolgt, von allen sei nur eine Facialisparalyse vollkommen geblieben.

Barth (Leipzig) will noch auf einen anderen wichtigen Punkt aufmerksam machen. Der Facialiscanal sei am Knie oberhalb des ovalen Fensters häufig offen und zwar so oft, dass das fast als das Normale angesehen werden könne. Häufig zuckt der Facialis deshalb beim blossen Tupfen oder Entfernen von Granulationen. Dass in Isolchen Fällen oft Lähmung eintritt, kommt hänfig daher, dass man zu fest tamponire. Er verbindet deshalb, sobald eine Lähmung sich zeigt, sogleich von Neuem und tam- 
ponirt lockerer. Man könne aus den angeführten Gründen nicht immer demjenigen, der eine Facialislähnung maeht, einen Vorwurf daraus machen.

Vohsen (Frankfurt) hat einen Fall beobachtet, bei dem nach 7 jährigem Bestehen einer Facialislähmung dureh Ohreiterung nach kurzer Faradisation noch Heilung erfolgte. In Betreff des Verlaufes des Facialis solle man auf folgenden Punkt achten: An dem Winkel oder Wulst, den der Facialis hinter dem ovalen Fenster macht, könne man schon sehen, wie er verläuft. Dieser Punkt sei aber leicht sichtbar, besonders wenn man nach Stacke operirt.

Kümmel (Breslau) bat eine nach 2 Jahren noeh bestehende Lähmung des Facialis 5 Jahre nach der Operation nicht mehr vorgefunden. Er glaubt nicht an eine günstige Beeinflussung durch den faradischen Strom. Er habe sich die Frage vorgelegt, weshalb der Facialis in einzelnen Fällen nicht zusammenheilt, während andere Nerven so leicht heilen, und sei der Ansieht, dass sich in solchen Fällen ein Knochenstückchen des Facialiscanales zwisehen beide Enden des Facialis einlagere. Man müsse deshalb in solchen Fällen den Facialis anfsuchen und die Enden freilegen, dann wïrde auch leicht eine Heilung eintreten.

Schw artze macht geltend, dass seine Absicht hier nur die gewesen sei, auf gewisse Anomalien im Verlauf des Facialis vom zweiten Knie abwärts aufmerksam zu machen. Dass nach Jahren, nachdem die Eiterung ausgeheilt war, nachträglich eine Lähmung noch zurückgegangen sei, hat er nicht erlebt. Er hält deshalb den Vobsen'schen Fall für ein Unicum.

Sehulze (Halle): Für die Annahme Kümmel's seheint eine Beobachtung ans der Halle'schen Klinik zu sprechen. Bei einem Fall, der 2 Jahre vorber von chirurgischer Seite erfolglos operirt war, bestand bei der Aufnahme vollständige Facialislähmung. Bei der jetzt vorgenommenen zweiten Operation wurde zwar kein Sequester im Facialiscanal gefunden, es wurde aber dicht am Facialis gearbeitet. Bald nach der Operation trat auffallende Besserung der Läbmung ein, welche 2 Jahre lang complet gewesen war.

Zaufal glaubt, dass die Chancen der Heilung dann die besten seien, wenn die Enden des Facialis möglichst nahe aneinander liegen.

Le u tert hält die Erklärung Kümmel's nicht für aus reichend. 
Buhe hat Gelegenheit gehabt, an einem anatomisehen Präparat den Facialis zu präpariren, wo im Leben 35 Jahre complete Facialislähmung bestanden hatte. Ein ganz dünner fadenförmiger Rest des Faoialis war erhalten, die beiden Enden versprengt in Knochenmassen eingebettet. (Nähere Beschreibung in diesem Arehiv.)

2. Walther Schulze (Halle): Ueber einen Fall von Empyem des Sacous endolymphaticus mit letalem Ausgange (mit Demonstration). (Ausführlich in diesem Arehiv.)

Diseussion:

Alexander (Wien) glaubt, dass dureh die frühzeitige Vornahme der Lumbalpunction in solchen Fällen früh genug die Entseheidung getroffen werden könne, $o b$ es sich um Meningitis oder um einen Abscess des Saceus endolymphatious handle.

3. Walther Sehulze (Halle): Zur Symptomatologie der Leptomeningitis purulenta ex otitide. (Wird ausführlich in diesem Arehiv erscheinen.)

Discussion:

Leutert fragt, weshalb man keine Meningitis ohne Lumbalpunotion diagnostieiren solle. Man könne die Diagnose Meningitis sehr wohl auch ohne Zuhilfenahme der Lumbalpunetion aus dem abrigen Symptomencomplex stellen. Er habe die Lumbalpunction nicht empfohlen zur Stellung der Diagnose auf Meningitis, sondern zur Differentialdiagnose zwischen Meningitis und Sinuserkrankung.

Sehwartze glaubt doch, die Lumbalpunktion in jedem fraglichen Falle empfehlen zu sollen, weil man sich sonst doch leicht täuschen könne. Im Allgemeinen sei das Ergebniss derselben für die Diagnose entscheidend; freilich seien in der Halle'schen Klinik übrigens in letzter Zeit Fälle vorgekommen, wo in der Lumbalpunetionsflüssigkeit vermehrte Leukocyten sich fanden und doch keine diffuse Meningitis vorlag.

Barth macht die Lumbalpunction in allen zweifelhaften Fällen. Er hält sie jedoch nicht für entscheidend für sein Handeln, sondern legt ihr mehr wissensehaftliches Interesse bei. Er operire auch den schwersten Fall noeb, selbst wenn Zeichen der Meningitis vorlägen. Deshalb falle bei ihm das praktische Interesse für die Lumbalpunction fort. Die Hörpriufungen empfiehlt er in solchen Fällen doch ja recht sorgfältig vorzunehmen, man könne sich dabei zu leicht täuschen. 
Le utert glaubt, dass bei jeder Trübung der Cerebrospinalflüssigkeit Meningitis vorhanden sei. Es komme gar nicht darauf an, ob die Trübung durch Leukocyten bedingt sei oder nicht. Jede Trübung berechtige zu dem Schlusse, dass eine meningitische Reizung vorliege.

Schwartze. wendet sich gegen Barth, der jeden Fall auch mit ausgesprochener Meningitis noch operirt. Der Standpunkt scheine ihm besonders für die Privatpraxis doch nicht rathsam zu sein. Nichts schädige das Ansehen des Arztes mehr, als eine unglïcklich verlanfene Operation.

Alexander fübrt nach Politzer jede Trübung auf Meningitis zurück. Sie seien zu dem Resultat gelangt, dass die Gerinnungsfähigkeit des Liquors von besonderer Bedeutung sei. Die mikroskopische Untersuchung hält er nicht für genügend beweisend, es müssen in jedem Falle Culturen angelegt werden.

Bezold hält den Weber'schen Versuch in solchen Fällen für unzuverlässig, besonders wenn der Patient Schmerzen auf dem zu prüfenden Ohre hat. Wenn Töne von a' aufwärts nicht mehr gehört werden, wird auch Sprache nicht mehr gehört. Weshalb prtife man nicht in solchen Fällen $a^{\prime}$ in der Luftleitung? Das sei ein wiehtiges Zeichen. Die Knochenleitung sei ganz unzuverlässig.

Barth steht auf dem Standpunkte, dass er sowohl Privatpatienten wie klinische in gleicher Weise behandle. Man müsse nur die Angehörigen in der riohtigen Weise vorbereiten. Ihm sei einmal ein Patient vollständig bewusstlos auf den Operationstisch gebracht, er eröffnete einen Hirnabscess und der Patient griff sehon auf dem Operationstisch nach dem Handtuch, um sich den Sehweiss abzuwischen.

Leutert glaubt nicht, dass Dehiscenzen im Tegmen tympani einen leichtern Uebergang der Entzïndung anf die $\mathrm{Me}$ ningen ermögliehen, bei Kindern seien dieselben sehr häufig, und doch sei bei ihnen die Gefahr nicht grösser. Er meint, die Auseinandersetzungen Schulze's über die Verschiedenartigkeit der Lumbalflùssigkeit verwirren die Auffassung.

Sehulze (Schlusswort) behauptet, dass in den erwähnten Fällen die congenitalen Dehiscenzen im Tegmen zweifellos den Uebergang der Eiterung auf die Schädelhöhle vermittelt haben, wie durch die genaue anatomische Untersuchung nachgewiesen wurde. Er will in denselben nicht etwa eine Prädilection für das Zustandekommen intracranieller Complicationen erblicken, 
möchte dieselben aber als nicht so ganz harmlos, wie neverdings von anderer Seite behauptet, hinstellen.

Herrn $B$ arth gegenüber betont der Vortragende, dass dic Hörprüfung in dem von ihm beobachteten Falle von Labyrwotheiterung: mit allen Cautelen ausgeführt worden sei, und dass der damalige Zustand der Patientin die Annahme einer Tsischung nicht zulasse.

Bezüglich der Lumbalpunction hält $\mathrm{S} \in \mathrm{h}$ u l d daran fest, dass weder der makroskopische Befund der Trübung allein noch der mikroskopische Nachweis der Vermehrung der Leukocyten in allen Fällen zur Diagnose der diffusen Meningitis purulenta genügt, sondern dass nach neueren Erfahrungen wahrscheinlich der Hauptwerth auf die bakterielle Untersuchung gelegt werden muss. Die Schwierigkeit des Nachweises der Bakterien ist oft dadurch bedingt, dass dieselben durch die Einwirkung des Liquor cerebrospinalis in ihrer Virulenz und Vitalität geschädigt werden. Das Culturverfahren lässt nicht selten im Stich da, wo man im Ausstrich Mikroorganismen nachweisen konnte. Die Färbbarkeit der Bakterien ist manchmal sehr herabgesetzt. Vortragender hat wiederholt die Beobachtung gemacht, dass bei längerer Färbung (bis zu 1 Stunde) Bakterien nachweisbar waren, wo die Färbung von gewöhnlicher Daner negativ ansfiel.

Wenn Leutert eine Lücke in der Beweisführung des Vortragenden findet in Betreff des einen Falles, in welchem der Liquor deutlich getrubt war, ohne Bakterien und vermehrte Leukoeyten zu enthalten (Fall Heinike, dieses Archiv Bd. LIV. S. 71), so ist Schulze sich dieser Lücke vollständig bewusst, hebt aber hervor, dass selbst die von berufenster Seite ausgeführten Untersuchungen in diesem Falle keine Erklärung uber die Natur der Trübung zu geben vermochten.

Die Frage der Lumbalpunction sei jedenfalls noch lange nicht definitiv entschieden, und es bedürfe zu ihrer vollkommenen Klärung noeh eingehender Untersuehungen.

4. Alexander (Wien): Demonstration dreier Modellreiben zur Entwieklungsgeschichte und-Anatomie des Gehörorganes.

I. Entwicklungsgeschichtliche Serie:

12 Modelle nach Wachsplattenmodellen des Ohrlabyrinthes

II. Anatomische Serie:

3 Modelle zur postembryonalen Entwicklung des Ohrlaby- 
134 XIIl. SCHULZE u. BUHE, 74. Versamml. deutsch. Naturf. u. Aerzte.

rinthes des Menschen nach Originalpräparaten des häntigen Labyrinthes und Metallausgüissen.

III. Ein zerlegbares Mittelohrmodell in 30 facher Vergrösserung lin.

Anwesend die Herren: Alexander-Wien, Alt-Wien, A nton-Prag, Band ler-Prag, Barth-Leipzig, Bauer-Prag, Bernatzky - Neisse, Berthold-Königsberg, Bezold-München, Biehl-Wien, Breitung-Coburg, Britz-Bad Reichenhall, Brüg gelmann-Berlin, Buhe-Halle a.S., Bunzlfedern-Prag, Eger-Dresden, Gomperz-Wien, Grunert-Halle a. S., Gutzmann-Berlin, Habermann-Graz, Heymann-Berlin, HnilitschkaNeudeck bei Karlsbad, H offmann-Dresden, Hópmann-Köln, ImhoferPrag, Joel-Görbersdorf, Jürgensmeyer-Bielefeld, Katz-Berlin, Ki ku c h i-Japan, K o ch - Schöneberg, K rez-Reichenhall, K ü gle - Schweidnitz, Krausha ar-Berlin, Krull-Güstrow, Kümmel-Breslan, Lentert-Giessen, Löwe-Berlin, Löwy-Karlsbad, Messem-New York, Müller-Karlsbad, Ortenau-Reichenhall, Piffl-Prag, Placzek-Iglau, Pollack-Wien, Rohde-Wien, Schmidt-Odessa, Schubert-Nürnberg, SchwartzeHalle a.S., Walther Schulze-Halle a.S., Steiner-Prag, UrbantschitschWien, Voh sen-Frankfurta. M., W e is s e n berg-Kolberg-Nervi, Wittma a ck Breslau, Za ufal-Prag, Zi mmermann-Dresden. 